\title{
MAPS AND FIGURE
}

\section{MAPS}

I Jiangnan in the Qing

2 Market Towns Attacked in the Zhaowen Tenant Uprisings of I 842 and I 846

3 The Lake Tai Region, ca. I850

4 Songjiang Prefecture and Taicang Department, ca. 1850

5 Jiangnan in the Republican Period

\section{FIGURE}

Prices of Paddy Land Sold in Yuanhe and Jiaxing, I797-I843 



\section{Rents, Taxes, and Peasant Resistance}

The Lower Yangzi Region, 1840-1950 
